\section{The Faculty of Accident and Emergency Medicine Annual Scientific Conference 2004}

\section{A1 USE OF A FORENSIC TECHNIQUE TO DETECT OCCULT BLOOD CONTAMINATION OF EMERGENCY DEPARTMENT AND AMBULANCE TRAUMA EQUIPMENT}

J. Lee, M. Levy, A. Walker.

Blood borne viruses are a recognised threat to health professionals involved in emergency care. Hepatitis B can remain viable in dried blood for 2 weeks and whilst health care professionals are protected by immunisation against this threat most patients are not. Previous studies have shown dental, radiological, and anaesthetic equipment, clean in appearance, to be contaminated with blood. This aim of this study was to determine whether equipment used in ambulances and Emergency Departments in the UK for the management of trauma victims was contaminated with blood.

Equipment from the emergency department of four hospitals (2 trusts) and the ambulances of two separate ambulance services will be tested, including 'spinal' boards, hard collars, head blocks, box splints/ inflatable/Thomas splints, blood pressure cuffs, pulse oximeter probes, tourniquet's, stethoscope heads, trolleys, ultrasound probes, laryngoscope blades, and bougies. Only equipment considered clean and ready for patient use will be tested. If the equipment is visibly blood stained, testing will not be performed. Equipment will be tested using the KastleMeyer technique, which is a highly specific forensic technique used by police to identify occult blood. Preselected areas for each type of equipment will be tested. The areas chosen are those considered by the authors as those most likely to come into contact with the patient.

Results will show the extent of blood contamination, type of equipment most frequently contaminated and highlight areas of equipment most frequently contaminated. Prehospital and hospital equipment contamination will be compared using Fisher's exact test or $\chi^{2}$ test.

\section{A2 NEW SCORING SYSTEM FOR CHILDREN WITH MENINGOCOCCAL DISEASE - AMPS (ANTRIM MENINGOCOCCAL PLACEMENT SCORE)}

J. Acheson, M. G. Jenkins, C. MaCleod, C. Patterson.

Introduction: Meningococcal disease mortality rates remain high and outcome is heavily dependant on early recognition, prompt treatment, and appropriate placement.

Objectives: Could those children with meningococcal disease, who will require intensive care admission, be identified quickly?

Design: Retrospective case note study of 88 cases in children who presented to Antrim Area Hospital. The cases were identified through Public Health notification of meningococcal cases over a three year period.

Method: Using the raw data, category variables underwent chisquared testing and continuous variables underwent t-tests. Multi logistic regression was performed to examine the independent effect of each significant variable. Using the co-efficients from logistic regression a new scoring system was produced which we have called the AMPS score (Age, Mentation, Perfusion, Shock). Scores were then calculated for this new system and for the Glasgow Meningococcal Septicaemia Prognostic score (GMPSS), which is presently used in this hospital. ROC curves were calculated for the two scoring systems and compared. The sensitivity and specificity for ICU admission was then calculated for each separate score in the AMPS system. The score with the highest percentage in both sensitivity and specificity was taken as an indication for possible ICU admission.

Results: The AMPS score consists of four variables; age, disturbed consciousness, capillary refill time, and peripheral vasoconstriction. The area under the ROC curve was greater for the AMPS score $(0.97)$ compared to the GMPSS (0.94). A score greater than 7 in the AMPS score had the highest percentage in both sensitivity (89.2\%) and specificity (93\%).
Conclusion: A score greater than 7 in the AMPS score could be used as an indication for possible ICU admission. This simple clinical score shows potential but will need validation.

\section{A3 COMPARISON OF THE PHARMACOKINETIC AND ANALGESIC PROFILES OF INTRANASAL AND INTRAVENOUS DIAMORPHINE IN CHILDREN}

S. Brennan. Specialist Registrar, Royal Hospital for Sick Children, Edinburgh S. Kidd. Staff Grade, A\&E, Royal Hospital for Sick Children, Edinburgh T. Beattie. Consultant, A\&E, Royal Hospital for Sick Children, Edinburgh R. Steven. Biochemist, Department of Child-life and Health, University of Edinburgh R. Minns. Head of Department, Department of childlife and health, University of Edinburgh

Background: The use of intranasal (IN) diamorphine for the treatment of acute paediatric pain is growing in popularity. However, the more invasive intravenous (IV) route is the current recommended gold standard. There has been no direct comparison of either the pharmacokinetic or analgesic profiles of these routes. Diamorphine is rapidly converted to morphine, a major biologically active metabolite $\left(t^{1 / 2} 9\right.$ minutes).

Objectives: To generate new pharmacokinetic data for diamorphine administered by the IN route. To compare this directly to IV data and pain scores collected in parallel.

Methods: Children aged 3-13 years attending a city centre paediatric A\&E department with clinical fracture of long bone had an IV cannula sited. Following scoring by 'Wong-Baker Faces' pain score at each time, blood samples were drawn at $0,2,5,10,20,30,60$ minutes post diamorphine administration $(0.1 \mathrm{mg} / \mathrm{kg}$ both routes). Blood was spun and frozen prior to analysis by solid phase ${ }^{125}$ radioimmunoassay.

Results: Significant differences in plasma morphine concentration were found at all times except baseline (Mann-Whitney-U). No significant correlations between plasma morphine levels and decrease in pain score were found at any time (Spearman's rank correlation $p>0.25$ ). No significant difference between groups was found for change in pain score at any time (Mann-Whitney-U, $p>0.2$ ).

\begin{tabular}{|c|c|c|c|}
\hline & IV group $n=12$ & IN group $n=12$ & Mann-Whitney-U \\
\hline & Median (range) & Median (range) & $\mathrm{p}$-value \\
\hline Age (years) & $9(4-12)$ & $7.5(4-12)$ & 0.69 \\
\hline Gender ( $m: f$ ) & $1: 1$ & $1: 2$ & 1.0 \\
\hline Weight (kg) & $33.0(16-43)$ & $30.1(19-59)$ & 0.98 \\
\hline Dose (mg) & $3.3(1.6-4.2)$ & $3.0(1.8-5.8)$ & 0.95 \\
\hline $\begin{array}{l}\text { Baseline pain } \\
\text { score }\end{array}$ & $2.0(1-6)$ & $2.0(1-6)$ & 0.49 \\
\hline
\end{tabular}

Conclusions: It has thus far been assumed that the IN route is almost equivalent to the IV route for diamorphine but we have shown a significantly attenuated and delayed peak in plasma morphine levels following IN administration. This suggests that further evaluation of pharmacokinetics may indicate an alteration in IN dosage. Pain scores are similar in each group, and this may be because of either the limitations of pain scoring, placebo effect, or analgesia from the direct effect of diamorphine or another metabolite. Further comparison of the analgesic profiles of diamorphine following IN and IV diamorphine are required.

\section{A4 ORAL OR TOPICAL ANALGESIA FOR CORNEAL ABRASIONS: A RANDOMIZED CONTROLLED TRIAL}

A. Newton, R. Rajendram, T. Even. Accident \& Emergency Department, John Radcliffe Hospital, Headley Way, Headington, Oxford, Oxfordshire, OX3 9DU

Methods: A prospective, double-blinded, randomized, controlled trial of 95 adult patients who presented with a diagnosis of traumatic corneal 


\section{A3 Table 2}

\begin{tabular}{|c|c|c|c|c|c|c|c|}
\hline $\begin{array}{l}\text { Minutes post diamorphine } \\
\text { dose }\end{array}$ & 0 & 2 & 5 & 10 & 20 & 30 & 60 \\
\hline $\begin{array}{l}\text { IN group Plasma } \\
\text { morphine(ngml }{ }^{-1} \text { ) } \\
\text { Median (IQ range) }\end{array}$ & $0.63(0.5-0.82)$ & $2.3(0.65-4.3)$ & $7.2(2.4-11.8)$ & $9.5(4.2-14.4)$ & $10.3(5.6-11.5)$ & $9.1(4.8-11.9)$ & $9.0(3.8-10.5)$ \\
\hline $\begin{array}{l}\text { IV group Plasma } \\
\text { morphine(ngml }{ }^{-1} \text { ) } \\
\text { Median (IQ range) }\end{array}$ & $0.51(0.28-0.89)$ & $31.0(21.8-56.1)$ & $26.8(22.6-41.9)$ & $21.9(19.6-38.8)$ & $19.6(15.4-21.7)$ & $15.3(13.3-17.4)$ & $13.5(9.6-17.9)$ \\
\hline$M-W-U$ p-value & 0.46 & $<0.001$ & $<0.001$ & $<0.001$ & 0.001 & 0.002 & 0.007 \\
\hline $\begin{array}{l}\text { IN group Median } \\
\text { 'Faces' score }\end{array}$ & 5 & 4 & 2 & 2 & 1 & 2 & 1 \\
\hline $\begin{array}{l}\text { IV group Median } \\
\text { 'Faces' score }\end{array}$ & 6 & 3 & 2 & 1 & 1 & 1 & 1.3 \\
\hline
\end{tabular}

abrasion to the Eye Casualty or the emergency departments of two district general hospitals. Patients were randomised to receive either oral diclofenac with placebo eye drops, or oral placebo with diclofenac eye drops. Primary outcome measure was pain recorded on a $100 \mathrm{~mm}$ visual analogue scale (VAS). Additional outcome measures included headache, foreign body sensation, light sensitivity, and sleep disturbance, each recorded on a four-point scale. Outcome measures were recorded by the patients at time of presentation, after each subsequent 6 hours for the first 24 hours, and finally at 48 hours after presentation. After 48 hours patients returned the study forms by post.

Results: Of the 95 patients recruited during the study period, 59 (62\%) returned the study forms. Of these, $34(57.6 \%)$ had diclofenac administered orally and $25(42.6 \%)$ topically. 49 patients correctly marked the visual analogue pain scale. Mean pain score was lower for patients using topical diclofenac, at 6 hours $(33.4 \mathrm{~mm} \vee 45.0 \mathrm{~mm}$, $\mathrm{p}=0.0708$ ) and at 12 hours ( $24.4 \mathrm{~mm} \vee 36.2 \mathrm{~mm}, \mathrm{p}=0.0767)$. Topical diclofenac was associated with lower foreign body sensation at 12 hours $(p=0.0153)$ and 18 hours $(p=0.0132)$. At 12 hours, topical diclofenac was also associated with lower light sensitivity $(p=0.0589)$ and sleep disturbances $(p=0.0590)$

Conclusions: In the management of traumatic corneal abrasion, better pain relief is achieved with topical administration of diclofenac. Symptoms of foreign body sensation, light sensitivity and sleep disturbance are also better controlled by topical diclofenac

\section{A5 PAEDIATRIC CARDIOPULMONARY RESUSCITATION: ONE HAND OR TWO?}

A. G. M. Stevenson, J. McGowan, C. A. Graham. Emergency Department, Southern General Hospital, Glasgow, Scotland, UK J. McGowan. Resuscitation Department, Southern General Hospital, Glasgow, Scotland UK A. L. Evans. Clinical Physics \& Bioengineering, Southern General Hospital, Scotland, UK C. A. Graham. A \& E Medicine Academic Unit, Chinese University of Hong Kong, Hong Kong

Aim: Current guidelines for chest compressions in cardiopulmonary resuscitation (CPR) advocate a one handed technique in children (1-8 years old) and a two handed technique in adults ( $>8$ years old). Variability in victim size or rescuer strength may require the use of two handed techniques for CPR in younger children. No previous study has examined whether the two techniques generate different compression pressures. This study aimed to assess the relative difference in intrathoracic compression pressures generated by one handed and two handed techniques in a paediatric model.

Methods: Randomised crossover design. Subjects (nurses, doctors, and medical students in an urban emergency department) performed chest compressions on an adapted paediatric resuscitation mannequin connected to a pressure transducer and computer. Subjects were randomised to the one handed or two handed technique for the first minute. Subjects performed one minute of chest compressions using one technique and then the other after a one minute rest period. Subjects' preference for one or two handed compressions was then determined. The pressures generated were compared using the paired t-test.

Results: 30 subjects (9 male, 21 female). Mean chest compression pressure was $86.56 \mathrm{mmHg}$ for two handed compressions and $75.14 \mathrm{mmHg}$ for one handed compressions, $p<0.001$. Average peak compression pressure was $133.50 \mathrm{mmHg}$ for two handed compressions and $116.83 \mathrm{mmHg}$ for one handed compressions, $p=0.001 .29 / 30$ found the two handed technique easier to perform.

Conclusion: Two handed chest compressions are easier to perform on a paediatric resuscitation mannequin and produce significantly higher mean and peak compression pressures. Further work is required to determine which technique produces improved clinical outcomes.

\section{Winner of the Rod Little Prize Session}

\section{A6 THE CHALICE RULE FOR MINOR HEAD INJURY IN CHILDREN: FROM THE CHILDREN'S HEAD INJURY ALGORITHM FOR THE PREDICTION OF IMPORTANT CLINICAL EVENTS, THE CHALICE STUDY}

J. Dunning, J-P. Lomas, J. Batchelor, K. Mackway-Jones. Department of Emergency Medicine, Manchester Royal Infirmary, UK D. Lloyd, H. Carty, B. Phillips. Department of Emergency Medicine, Alder Hey Hospital, Liverpool, UK J. P. Daly. Manchester Computing, Manchester, UK F. Lecky. Department of Emergency Medicine, Hope Hospital, Manchester, UK P. Brennan. Department of Emergency Medicine, Sheffield Children's Hospital, UK A. Waters. Department of Emergency Medicine, Countess of Chester Hospital, Chester, UK S. Southworth. Department of Emergency Medicine, Stepping Hill Hospital, Manchester, UK S. Kumar. Department of Emergency Medicine, Bury General Hospital, Bury, UK K. Ali. Department of Emergency Medicine, Royal Oldham Hospital, Oldham, UK D. Ryan. Department of Emergency Medicine, Wythenshawe Hospital, Manchester, UK L. Duane. Department of Emergency Medicine, Booth Hall Children's Hospital, Manchester, UK

Background: Half of all patients presenting to emergency departments are children, but while there are several large, well conducted, studies in adults that allow accurate selection of high risk patients for CT scanning, no such study has derived a rule for children. We sought to perform a prospective multicentre diagnostic cohort study to provide such a rule.

Methods: All patients presenting to the emergency departments of 10 hospitals in the Northwest of England with any severity of head injury were recruited into our study. A tailor-made proforma was used to collect data on over 40 clinical correlates for each patient. All patients who had positive pathology on CT scanning were identified. Recursive partitioning was used to create a highly sensitive rule for the prediction of significant intracranial pathology.

Results: 22,772 patients were recruited over a $2 \frac{1}{2}$ year period. $65 \%$ of patients were male and $56 \%$ were under 5 years of age. 281 patients had a positive CT scan, 137 patients had a neurosurgical operation, and 15 patients died. The CHALICE rule was derived that has a sensitivity of $98.4 \%(96.4 \%-99.6 \%)$, and a specificity of $86.9 \%(86.5 \%-87.4 \%)$, and requires a $14 \%$ CT scan rate. This rule was superior to actual practise during the trial and to current recommended guidelines in the UK.

Conclusion: We have successfully derived a highly sensitive clinical decision rule for the identification of children after head injury who should undergo CT scanning. This rule has the potential to improve and standardise the care of children presenting with head injuries. 


\section{Free Paper Session 1}

\section{A7 A CLINICAL PREDICTION RULE TO IDENTIFY EMERGENCY ROOM ATTENDEES WITH SEVERE ACUTE RESPIRATORY SYNDROME (SARS) DURING AN OUTBREAK}

T. H. Rainer, G. M. Leung, F-L. Lau, I. O. L. Wong, A. Tong, T-W. Wong J. H. B. Kong, A. J. Hedley, T-H. Lam, for Hospital Authority SARS Collaborative Group (HASCOG). Accident and Emergency Medicine Academic Unit, Trauma and Emergency Centre, Prince of Wales Hospital, Shatin, Hong Kong

Background: The resurgence of SARS remains a distinct possibility Accurate, objective models of triage and diagnosis could help physicians and public health authorities assess patients' risks and improve decisions about isolation and treatment in hospital.

Methods: From analysis of data on 1,274 emergency room attendees (derivation cohort) during Hong Kong's 2003 epidemic, we developed a clinical prediction rule to identify patients with SARS using a two-step process. We validated the predictive accuracy of this rule internally using the bootstrap method and externally on 1,375 patients attending another emergency room (validation cohort). The prediction rule assigns points based on history, physical examination, and simple investigations on presentation to the emergency room, obtained from multivariable logistic regression equations by using a regression coefficient-based scoring method. The outcome measure was a final diagnosis of SARS confirmed by World Health Organization laboratory criteria.

Results: Predictors for SARS on history (step 1) included previous contact with a SARS patient, presence of fever, myalgia, chills or rigor, and malaise. Older age ( $\geqslant 65$ years) and the presence of sputum production were inversely related to a diagnosis of SARS. In step 2 , simple investigative findings such as a positive chest X-ray (haziness or pneumonic consolidation), low lymphocyte, and low platelet counts were associated with a higher probability and a high neutrophil count with a lower probability of SARS. In the derivation sample, the observed SARS incidence rate was $4.3 \%$ for those assigned to the low-risk group (in steps 1 or 2), $18.8 \%$ (risk score $=-1$ to 1 ) for quartile one in the high-risk group, $55.7 \%$ (risk score $=2$ to 3 ) for quartile two, $66.7 \%$ (risk score $=4$ ) for quartile three, and $80.2 \%$ (risk score $\geqslant 5$ ) for quartile four. We observe similar gradations of risk in the validation cohort. This rule achieved a sensitivity of $0.94(0.90)$ and a specificity of $0.61(0.40)$ in the derivation (validation) cohort. The areas under the receiver operating characteristic curve were 0.88 and 0.84 in the derivation and validation samples respectively.

Conclusions: Our findings suggest that a simple model using clinical data at the time of presentation to an emergency room during an acute outbreak predicted SARS incidence and provided good diagnostic utility.

\section{A8 EARLY CHANGES IN COAGULATION FOLLOWING SEVERE INJURY}

M. Heron, T. Coats. Royal London Hospital, UK

Introduction: Coagulation is a vital part of the physiological response to injury. Disturbance of coagulation is common and is associated with increased mortality. Despite the importance of coagulation the early changes (within the first 4 hours of injury) in the haemostatic system have been poorly defined. This study was designed to describe the early changes in the coagulation system following injury.

Methods: A convenience sample of 50 injured patients was studied. Four blood samples were taken from each patient. The first sample was taken as early as possible in the prehospital phase, the second on arrival at the Emergency Department, followed by further samples at 2.5 and 5 hours after injury.

Measurement was made of: (1) activation of thrombosis (Tissue factor); (2) amount of thrombin generation (Fragment 1.2 and TAT); (3) inhibitors of thrombin generation (Antithrobin III and Protein C); (4 activation of fibrinolysis (TPA); (5) inhibition of fibrinolysis (PAl); and (6) amount of fibrinolysis (D-Dimer). Measurement was also made of the markers of inflammation ILI, IL6, and TNF. A conventional coagulation screen was also taken.

Results: The median ISS of the patients was 26 . There was a large immediate rise in Tissue Factor, which had almost disappeared by the time the patient had reached the Emergency Room. This resulted in an intense thrombotic response. This was followed by a sustained fibrinolytic phase, with ongoing thrombosis in some patients. The prehospital INR was normal, but there was increasing abnormality with time. In contrast, whole blood coagulation (thromboelastogram) was abnormal in $52 \%$ of patients on arrival in the resuscitation room. There was no correlation between the amount of tissue factor released and the Injury Severity Score.

Conclusion: This is the first description of the early changes in coagulation after injury. The intensity and speed of the tissue factor response is surprising, and suggests an origin in monocytes rather than endothelium. Several potential therapeutic targets are identified. Despite the large disturbance in coagulation the conventional coagulation screen remains normal until a late stage.

\section{A9 CARDIAC OUTPUT IN THE EMERGENCY DEPARTMENT USING THORACIC ELECTRICAL BIOIMPEDENCE MEASUREMENT - A FEASIBILITY STUDY}

\section{T. Coats, B. Loryman, F. Patel.}

Background: Cardiac Output measurement by Thoracic Electrical Bioimpedence is an old technique, which has recently been adapted to remove movement artifact - making this a potentially useful technique in the Emergency Room. The technique measures changes in thoracic impedance caused by the blood flow up and down the aorta with each heartbeat. The pattern of change in impedance is analysed and related to cardiac output. The application of this method to the emergency patient has not been well studied. There is no data on which to base sample size calculations and it is not known if a good signal can be measured in the ED. The aims of this study were (1) to establish the feasibility of TEB recording in the ED and (2) to establish the distribution of Cardiac Output variables measured in the ED.

Methods: Patients presenting to the ED with breathlessness (RR>20) were included in the study. After patient consent or relative assent was obtained the recording electrodes were placed and a 10 minute period of continuous TEB cardiac output recording was taken.

Results: 26 patients were studied. In two patients no stable signal could be obtained, and in a further three patients only a small proportion of good signal ( $<20 \%$ of the recording period) could be obtained. In 21 of the patients $(80 \%)$ enough signal could be obtained to derive a cardiac output measurement.

The Cardiac Index of the 26 patients ranged from 1.89 to 8.27 , the reading of 8.27 being an outlier (this patient was breathless due to anaphylaxis). The data was normally distributed (Kolmogorov-Smirnov test for normal distribution) with a mean of $3.6,95 \% \mathrm{Cl}$ from 3.0 to 4.3 , and standard deviation of 1.5 .

Conclusions: TEB is a feasible technique in the Emergency Room, with about $80 \%$ successful recording. This preliminary data from a population of breathless patients gives us the data on which to base sample size calculations for future studies.

\section{A10 UTILITY OF INITIAL CARDIAC TROPONIN AND ISCHAEMIA MODIFIED ALBUMIN MEASUREMENTS FOR RAPID RULE OUT OF MYOCARDIAL INFARCTION}

S. Goodacre, P. O. Collinson, D. C. Gaze, K. Bainbridge, F. Morris, B. Morris, A. Price. Accident \& Emergency Department, Northern General Hospital, Herries Road, Sheffield, S5 7AU

Objective: To assess if the combination of cardiac troponin and ischaemia modified albumin (IMA $\left.{ }^{T M}\right)$ can be used for very early exclusion of acute myocardial infarction (AMI)

Methods: Patients attending the ED with chest pain, undiagnosed by initial clinical assessment, ECG and chest radiograph, had blood drawn shortly after arrival. They then underwent AMl rule-out (using a combination of $\mathrm{CK}-\mathrm{MB}$ (mass) gradient over $>2$ hours and cardiac troponin $\mathrm{T}(\mathrm{cTnT})$ measurement $>6$ hours after pain onset), exercise treadmill testing, and follow-up at 72 hours from presentation for further cTnT measurement. A final diagnosis of $\mathrm{AMI}$ was made according to ACC/ECS criteria using a cut off of $0.03 \mu \mathrm{g} / \mathrm{L}$ cTnT at 6 or 72 hours. A serum aliquot from the initial blood sample was frozen and subsequently analysed for IMA ${ }^{T M}$. If ${ }^{T T n T}$ had not been measured on the original sample, $c T n l$ was measured $(n=189)$. Troponin was determined to be positive if it exceeded a threshold of $\geqslant 0.03 \mu \mathrm{g} / \mathrm{L}$ and initial IMA was positive if it exceeded a threshold of $85 \mathrm{U} / \mathrm{L}$. These results were then compared to the final diagnosis.

Results: Samples were collected from 539 patients $(335$ male, 204 female, median age 51.9 years). Cardiac troponin or IMA ${ }^{\text {TM }}$ was elevated in the admission sample of all patients with a final diagnosis of AMI $(n=46)$. IMA ${ }^{T M}$ alone was elevated in $5 / 46$, cardiac troponin alone in $19 / 46$ and both were elevated in 22/46. AMI was excluded (both tests negative) in 171/494 (35\%) patients. 
Conclusion: Admission measurement of cardiac troponin plus IMA ${ }^{T M}$ could be used to provide immediate triage and rule-out $A M I$ in one third of patients presenting with acute, undifferentiated chest pain.

\section{A11 WHICH CLINICAL FINDINGS ARE USEFUL IN EVALUATING THE RISK OF A FRACTURE AFTER PAEDIATRIC WRIST TRAUMA}

A. Webster, D. Walker, S. Goodacre, D. Burke.

Background: Clinical decision rules have been demonstrated to reduce uncertainty in medical decision making. Using a minimum of three factors based on history taking, examination and simple investigations they can enable clinicians to make safe decisions while using resources effectively. Are clinical findings following trauma to the wrist useful in predicting the likelihood of a fracture in the paediatric population?

Methods: This prospective cohort study was carried out in the Emergency Department at Sheffield Children's Hospital. Eligible patients aged between 3 and 16 years presenting following wrist trauma were recruited. A structured data collection form was completed by the clinician prior to any request for an $x$-ray. The gold standard outcome was radiological interpretation; in cases of doubt AW reviewed the departmental notes and any orthopaedic evaluation to make a final decision. Statistical analysis was carried out using SPSS version 11.5. Univariate analysis was performed using $\chi^{2}$ for categorical variables and Mann Whitney $U$ test for continuous data.

Results: There were 220 patients recruited, with 104 (47.3\%) fractures diagnosed. $88.2 \%$ of all patients received an $\mathrm{x}$-ray. 10 clinical variables were evaluated, abnormal grip strength, focal swelling, reduction in supination/pronation, distal radial tenderness, and clinical suspicion were all significantly associated with a final diagnosis of fracture after univariate analysis $(p<0.1)$

Absence of radial tenderness reduced the odds of a fracture five-fold while normal supination/pronation and absence of focal swelling both roughly halved the risk of fracture.

Conclusion: Following an injury to the wrist, absence of radial tenderness was found to be the best discriminating clinical finding for a normal wrist x-ray. However, nine out of 104 fractures would still have been missed including three scaphoid fractures. We suggest that clinical judgement is still important in deciding the need for an x-ray following paediatric wrist trauma.

\section{A12 PATIENT DEPENDENCY IN THE EMERGENCY DEPARTMENT: DO WE HAVE THE NURSES WE NEED?}

A. O'Brien, J. Benger. Academic Department of Emergency Care, Emergency Department, Bristol Royal Infirmary, Marlborough Street, Bristol BS2 8HW

Background: Nursing salaries are the largest single expenditure in the NHS, but the number and grade of nursing staff in a particular clinical area is often based on historical or arbitrary measures. In the emergency department (ED), staffing calculations based only on the number of patients fail to take account of the dependency, and hence the actual nursing workload, of these patients.

Objectives: We set out to use the previously validated Jones Dependency Tool (JDT) to establish current patterns in the dependency of patients attending the adult ED of an inner city teaching hospital, to better inform present and future decisions regarding nursing numbers and grade mix.

Methods: The JDT was used to prospectively collect data on the dependency of all patients attending the adult ED of the Bristol Royal Infirmary over a continuous 2 week period. The primary outcome measure was patient dependency assessed on arrival and departure, and at 4 and 8 hours after arrival if the patient was still in the ED. The location of the patient and triage category were also recorded. Data were analysed using descriptive statistics and chi-squared testing

Results: Data were collected on $48 \%$ of all patient attendances during the 2 week period. Analysis of the triage category of all patients compared to the triage category of those for which data had been collected suggested that this sample was representative. $10 \%$ of patients fell into the high or total dependency groups on arrival. Median length of stay for all patients was 3 hours and 5 minutes, with low and total dependency patients staying for shorter periods in the ED. Patients tended to become less dependent during their ED stay, and analysis of those individuals in which dependency unexpectedly increased was clinically valuable. The proportion of high and total dependency patients did not vary by day of the week, but was significantly increased overnight.

Discussion: The JDT can be used to collect reliable and informative dependency data in a busy working ED. These data inform both staffing and capacity planning, since dependency also predicts resuscitation room care. The proportional increase in high and total dependency patients overnight emphasises the weakness of basing staffing levels on patient numbers alone.

Conclusion: Patient dependency is one of the essential determinants of nursing grade mix, and can be used to predict workload, resource use and the optimal staffing levels that will provide safe and effective patient care. Dependency can be readily and repeatedly assessed, and we recommend this approach to other Emergency Departments.

\section{Free Paper Session 2}

\section{A13 VALIDATION OF ULTRASONOGRAPHY BY EMERGENCY DEPARTMENT PHYSICIANS IN THE SCREENING FOR ABDOMINAL AORTIC ANEURYSMS IN ACUTE CORONARY SYNDROME}

D. Monaghan, N. Collum, D. Hartin, R. McLaughlin, C. Martyn, J. Bowra, S. J. McGovern.

Background: Abdominal aortic aneurysm (AAA) has shared risk factors with coronary artery disease.

Ruptured AAA has a mortality of $>80 \%$, whereas elective repair mortality is $5-6 \%$.

A recent $\mathrm{BMJ}$ publication calls for a national screening programme to be established.

We present preliminary results of ED point of care screening for AAA in patients presenting with acute coronary syndrome (ACS).

Materials \& methods: A prospective cohort study from 26/11/03 to $17 / 06 / 04$. A convenience sample of patients aged $>50 \mathrm{yrs}$ attending the ED of the Ulster Hospital, Belfast with a working diagnosis of ACS were enrolled. If no exclusion criteria were present (Known AAA, unable or unwilling to provide informed consent, clinical condition precluding ultrasound (US)), the patient underwent a focused US for AAA (maximum diameter $>3 \mathrm{~cm}$ ). An Emergency Physician (EP) not involved directly with the clinical care performed and interpreted this. These EP's had received goal directed training in ED US prior to the study period.

A radiologist, blinded to the ED scan interpretation, reviewed all scans. Patients whose scan was reviewed as inadequate had radiology department US performed. Results were compared.

Results: 50 patients have been enrolled. Mean patient age was 71 years, with a male to female ratio of 3.2:1. Aortic diameter ranged from $1.4 \mathrm{~cm}$ to $4.9 \mathrm{~cm}$. All ED scans were adequate and showed $100 \%$ agreement with radiologist review.

Three $(6 \%)$ scans demonstrated AAA present measuring $3.3 \mathrm{~cm}$ to $4.9 \mathrm{~cm}$. These patients were referred for vascular opinion.

Conclusions: This study suggests that with minimal training, EP's can accurately assess aortic diameter in a population at increased risk. This point-of-care test also represents an accurate screening method for AAA and may have implications in the delivery of thrombolysis and earlier vascular referral.

\section{A14 A RANDOMISED TRIAL TO COMPARE THE EFFICACY AND SAFETY OF INTRANASAL LORAZEPAM AND INTRAMUSCULAR PARALDEHYDE IN THE TREATMENT OF CONVULSIONS IN CHILDREN}

S. Ahmad, E. Molyneux. Department of Paediatrics, College of Medicine, University of Malawi

Introduction: At the Queen Elizabeth Central Hospital (QECH), Blantyre, in Malawi, 75,000 children per annum visit the A\&E Department. Of these approximately 1000 patients per annum are gravely ill and are treated in the resuscitation area. Respiratory failure, shock, and coma due to pneumonia, gastroenteritis, severe malaria, bacterial meningitis, and septicaemia are the commonest conditions treated here. 300 of these very sick children presented as convulsions in 2003 to our department.

The use of intravenous lorazepam as first line therapy in acute childhood seizures where venous access has been obtained is widely accepted in Europe and North America. In the absence of venous access, rectal diazepam or paraldehyde is an effective alternative in both well and poorly resourced countries.

In Malawi paraldehyde is the first line of treatment and is given by the intramuscular route. It can be effective in stopping seizures and is relatively safe but is expensive, slow to administer, painful, and has the potential to cause injury and infection at the injection site. 
Although benzodiazepines have long been the mainstay of first line therapy for acute seizures, there remains insufficient clinical data as to the optimal first line non-intravenous drug of choice or mode of administration where venous access has not been established.

Methods: This is a prospective open randomised trial comparing the efficacy and safety of intranasal lorazepam to intramuscular paraIdehyde. The study will be carried out in the Paediatric Accident \& Emergency Department, QECH, Blantyre. Approximately 25 cases of acute seizures present to this department a month. We expect to reach our sample size (352) in 14 months (July 2004 - September 2005). This study has been approved by the College of Medicine Research \& Ethics Committee, University of Malawi (P.03/04/248)

Results: We propose to present preliminary data of this study, which wishes to address the urgent need to obtain randomised controlled data in treating acute tonic convulsions and convulsive status epilepticus in children using a drug and delivery system that is safe, effective and easy to deliver.

\section{A15 MORTALITY AFTER TRAUMA INTUBATION WITHOUT DRUGS IN SCOTTISH EMERGENCY DEPARTMENTS}

C. A. Graham. A \& E Medicine Academic Unit, Chinese University of Hong Kong, Hong Kong G. M. Wares. Department of Anaesthesia, Western Infirmary, Glasgow, Scotland, UK D. Beard. Scottish Trauma Audit Group, Royal Infirmary of Edinburgh, Scotland, UK P. T. Munro. Emergency Department, Southern General Hospital, Glasgow, Scotland, UK

Aim: Airway management is a core element of trauma care. Recent work has suggested that trauma patients who are intubated in the prehospital arena without drugs have a very poor prognosis. This study aimed to identify the outcome of trauma patients intubated without drugs in Scottish emergency departments (EDs).

Methods: Retrospective cohort study using prospectively collected data from the Scottish Trauma Audit Group between 1 January 1999 and 31 December 2002. Patients who were intubated were identified from the database and the number of patients who required drugs for intubation was determined. The mortality of those who were intubated with and without drugs was calculated. Statistical analyses: the chi square test was used for categorical data and the Mann Whitney $U$ test was used for non-parametric data (GCS, ISS, age)

Results: 24756 patients in the STAG database, 1503 intubations: 1244 with drugs and 218 with no drugs (41 unknown). No significant difference in the proportion of males $80.8 \%$ with drugs, $86.2 \%$ without, $p=0.57$ ) or median age (38 years with drugs, 36 without, $p=0.51$ ). Median GCS was 8 in the drugs group and 3 in the no drugs group $(p<0.001)$. Emergency physicians intubated a higher proportion of patients in the no drugs group $(50.7 \% \vee 21.4 \%, p<0.001)$. Median ISS was higher in the no drugs group $(30 \vee 25, \mathrm{p}<0.001)$ and mortality was also higher in the no drugs group $(75.7 \%$ v $30.2 \%, \mathrm{p}<0.001)$.

Conclusion: Trauma patients in Scottish EDs who are intubated without drugs have high mortality rates. Outcome is not universally fatal but morbidity is unknown and requires further research. There is a need to identify the underlying diagnoses in these patients to identify factors associated with survival or death.

\section{A16 CONTRIBUTION OF COCAINE USE TO CHEST PAIN AND MYOCARDIAL INFARCTION IN EMERGENCY DEPARTMENT PATIENTS}

T. Maric, S. O'Connor, J. A. Henry. Academic Department of Accident and Emergency Medicine, Imperial College, St. Mary's Hospital, London, W2 INY
Introduction: Cocaine is currently responsible for more United States Emergency Department visits than any other illicit drug. In the UK cocaine is the only drug to show a significant rise in misuse between 1994 and 2004. It commonly causes chest pain due to coronary vasospasm, and can also cause myocardial infarction. We wished to examine the incidence of cocaine related chest pain and myocardial infarction

Methods: With ethics committee approval, patients presenting with and without chest pain had a urine sample collected at presentation, labelled and stored. Outcomes were obtained by studying case records after 48 hours. Each chest pain patient was matched with a non-chest pain control. Patient stickers were then destroyed and samples were allocated a study number linking the sample to the established outcome but not to the patient's name, preserving anonymity. Urine samples were then tested for cocaine or metabolites, results recorded and samples discarded.

Results: Of the 587 patients with chest pain, $370(63 \%)$ were men and $217(37 \%)$ women. Cocaine was detected in the urine of 46 chest pain patients $(7.8 \%)$. Test and control groups differed $(p<0.004)$. One patient developed a myocardial infarction. Cocaine use was highest in those aged 18-30 years for both sexes and decreased with age. Cocaine positive chest pain patients were more likely to present in the evening or after midnight.

Discussion: As most patients will not admit to use of an illegal drug, routine testing would allow cocaine related ischaemic events to be identified more easily but remains controversial. Since management of cocaine-induced chest pain is different from other causes of chest pain, doctors should question chest pain patients about its use.

\section{A17 COMPARISON OF CIRCULATING PLASMA DNA AND S-100 PROTEIN CONCENTRATIONS IN PATIENTS WITH ACUTE STROKE}

T. H. Rainer, K. S. Wong, W. Lam, E. Yuen, N. Y. L. Lam, Y. M. D. Lo. Accident and Emergency Medicine Academic Unit, Prince of Wales Hospital, Chinese University of Hong Kong

Aim: This study aimed to compare the predictive value of early changes in circulating plasma DNA and S-100 protein in predicting mortality and morbidity in patients with stroke.

Methods: Patients with stroke-like symptoms who presented to an emergency department of a university hospital in Hong Kong were recruited in the study. DNA extracted from patients' plasma was analysed for the $\beta$-globin gene with a fluorescent-based polymerasechain-reaction test. S-100 protein concentrations were determined using an ELISA kit. The primary outcome measures were mortality and morbidity using the post-stroke Modified Rankin Score.

Results: Of 175 consecutive patients recruited to the study, 105 (60\%) had ischemic stroke, 29 (17\%) had intracerebral hemorrhage, and 41 (23\%) had negative neuroimaging. Plasma DNA concentrations $>1400$ kilogenome-equivalents/L have a sensitivity of $82 \%$ and a specificity of $69 \%$ for predicting six-month mortality. Plasma S-100 protein concentrations $>0.17 \mu \mathrm{g} / \mathrm{L}$ have a sensitivity of $70 \%$ and a specificity of $72 \%$ for predicting 6 month mortality. The differences between the areas under the Receiver Operator Characteristic curve for DNA and S-100 at discharge and for six mortality were $0.059(95 \% \mathrm{Cl}-0.174$ to 0.293$)$ and $0.078(95 \% \mathrm{Cl}-0.116$ to 0.271$)$ respectively, and for 6 month post-Rankin score $<2$ was $0.027(95 \% \mathrm{Cl}-0.081$ to 0.135$)$. The adjusted odds ratios for plasma DNA levels predicting 6 month mortality was $1.585(95 \% \mathrm{Cl} 1.052-2.389 ; \mathrm{p}=0.03)$, and for predicting 6 month post-Rankin score $>2$ was $1.819(95 \% \mathrm{Cl} 1.000-3.312 ; p=0.05)$.

Conclusion: Plasma DNA levels and S-100 protein levels predict mortality and morbidity equally well in patients presenting with stroke. 